\title{
Rate and Associated Factors of Post-Traumatic Stress Disorder in Elderly Survivors of Bombardment in Ilam City, Iran
}

\section{ART I C L E INF O}

\section{Article Type}

Descriptive Study

\section{Authors}

Rostamizadeh A. ${ }^{1} M S C$, Abolfathi Momtaz Y.*1 $P h D$, Foroughan M. ${ }^{1} M D$

How to cite this article
Rostamizadeh A, Abolfathi
Momtaz Y, Foroughan M. Rate
and Associated Factors of Post-
Traumatic Stress Disorder in Eld-
erly Survivors of Bombardment in
Ilam City, Iran. Iranian Journal of
War \& Public Health.2020;12(2):
125-132.

${ }^{1}$ Iranian Research Center on Aging, University of Social Welfare and Rehabilitation Sciences, Tehran, Iran

\section{*Correspondence}

Address: Iranian Research Center on Aging, University of Social Welfare and Rehabilitation Sciences, Koodakyar Street, Daneshjoo Boulevard, Velenjak Square, Tehran, Iran. Postal Code: 1985713834.

Phone: +98 (21) 71732256

Fax: +98 (21) 22180154

yabolfathi@gmail.com

\section{Article History}

Received: January 26, 2020

Accepted: May 18, 2020

ePublished: June 17, 2020

\section{A B S T R A C T}

Aims The psychological effects of war, such as post-traumatic stress disorder, may persist for years after the war. The aim of this study was to estimate the rate of post-traumatic stress disorder and its related factors in the elderly survivors of the bombing in Ilam.

Instruments \& Methods The present study was performed by retrospective cohort method on 227 elderly survivors of the imposed war living in Ilam City, Iran, in 2019 who were selected by multi-stage proportional random sampling. The NSESSS-PTSD instrument was used to measure post-traumatic stress disorder. Used descriptive and inferential statistics (independent t-test, ANOVA, and Correlation) for data analysis by SPSS 23 software.

Findings The mean age of the elderly participating in the study was $66.67 \pm 7.16$ years. 54.2\% of participants were female. There was a significant relationship between marital status, employment, witnessing injuries and deaths, age at wartime and gender, and post-traumatic stress disorder. There was a significant relationship between current chronic patients and post-traumatic stress disorder.

Conclusion $16.3 \%$ of the elderly survivors of the bombing of the Iran-Iraq war in Ilam have post-traumatic stress disorder.

Keywords PTSD; Elderly; Bombs

\section{I T A T I O N L I N K S}

[1] Evaluation of effectiveness of training ... [2] Post traumatic stress disorder ... [3] Prevalence of post-traumatic ... [4] Evaluation of post-traumatic stress ... [5] Post-traumatic stress ... [6] Posttraumatic stress disorder in older ... [7] Prevalence of post-traumatic ... [8] Life experiences of wives of ... [9] Effect of exposure to the traumatic ... [10] Demographic characteristics and post-traumatic ... [11] Investigating the impact of exposure ... [12] Research findings, limitations ... [13] Persisting posttraumatic stress disorder ... [14] The impact of posttraumatic stress ... [15] Differences among ethnic groups ... [16] A metaanalysis of risk factors for ... [17] The comparison of the quality of life of ... [18] Traumatic experiences and post-traumatic ... [19] Mental health condition and health ... [20] Stress management, history and ... [21] Determining the major stressful events ... [22] History of Defa-e-Moqadas in Ilam ... [23] Dimensional assessment of post traumatic ... [24] Evaluating the Persian translation of ... [25] Psychometric properties of the Turkish ... [26] Prevalence and risk factors of posttraumatic ... [27] Integrative testimonial therapy: An ... [28] Depression and PTSD in Pashtun ... [29] A comparison between loneliness and ... [30] Evaluation of PTSD in Sardasht survivors ... [31] How well can post-traumatic stress ... [32] Mental pathology based on ... [33] Kaplan \& Sadok psychiatric summary ... [34] Prolonged grief disorder, depression ... [35] Prevalence of posttraumatic stress ... [36] Prevalence of mental disorders ... [37] Posttraumatic stress disorder and ... [38] Post-traumatic stress disorder and ... [39] Effectiveness of biofeedback ... [40] Elevated serum lipids in veterans with ... [41] Abnormal serum lipid profile in Brazilian ... [42] Is stress levels linked to impaired ... [43] Effect of mental stress on serum ... [44] The role of psychosocial stress ... [45] Relationship between the prevalence ... [46] Prevalence of musculoskeletal disorders ... [47] The association between tinnitus ... [48] Identifying Physical health status ... [49] Post-traumatic stress disorder, social ... [50] Related factors to onset of post-traumatic ... 
و معمولاً شامل يك تهديد ادراكشده درباره زندكى يا تماميت

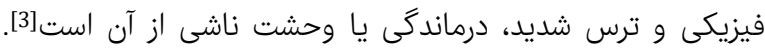

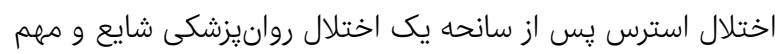

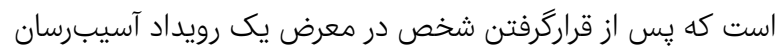

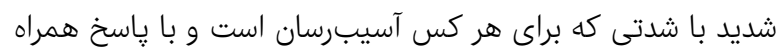

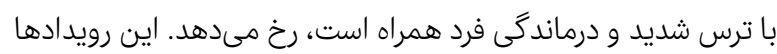

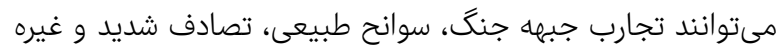

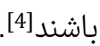

در اين اختلال نشانههاى مرضى روانشناختى به دنبال يك حادثه

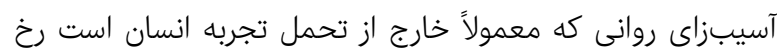

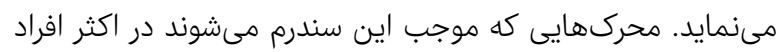

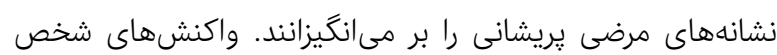

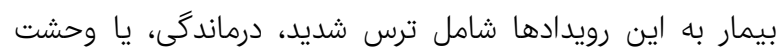

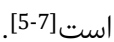

اختلال استرس يس إز سانحه يك اختلال اضطرابى شديد و وراني

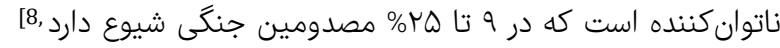

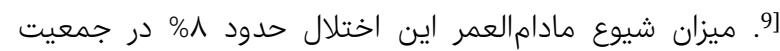

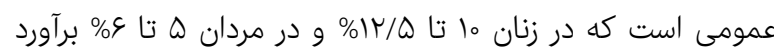

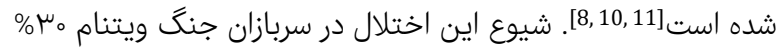
أس

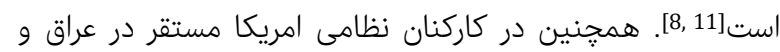

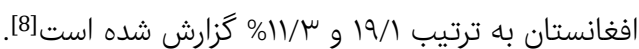

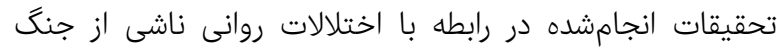

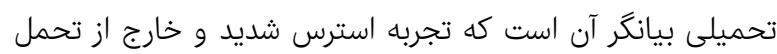

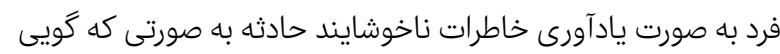

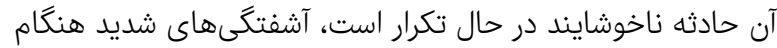

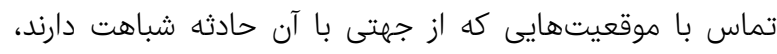

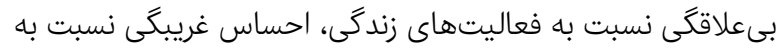

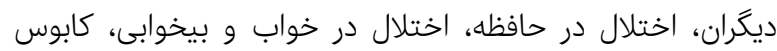

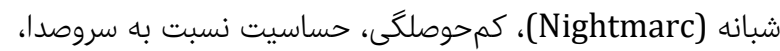
تحريكيذيرى، كاهش بازده كار، سردرد، فراموشى، اضطراب،

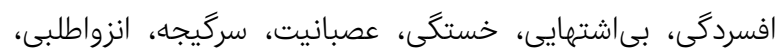

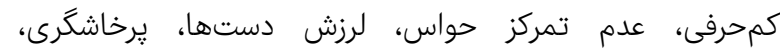

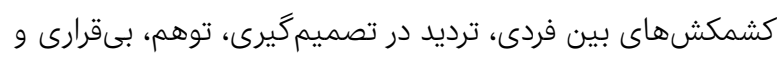

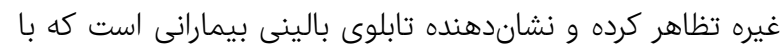

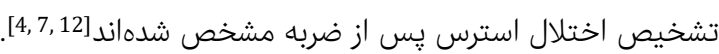

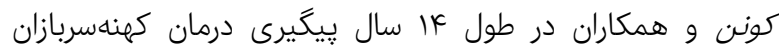

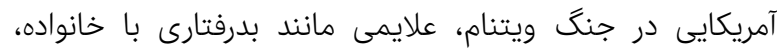

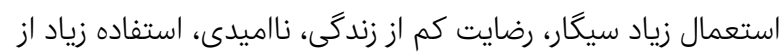

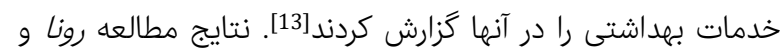

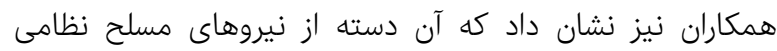

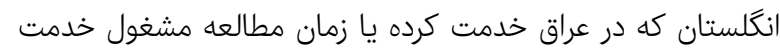

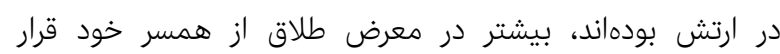

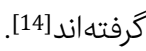

\section{ميزان و عوامل مرتبط با اختلال استرس یس از} سانحه در سالمندان بازمانده از بمباران شهر ايلام

افسانه رستمىزاده MSc

مركز تحقيقات سالمندى، دانشكاه علوم بهزيستى و توانبخشى، تهران، ايران

يداله ابوالفتحى ممتاز * مhن

مركز تحقيقات سالمندى، دانشًاه علوم بهزيستى و توانبخشى، تهران، ايران

MD مهيد فروغان

مركز تحقيقات سالمندى، دانشًاه علوم بهزيستى و توانبخشى، تهران، ايران

جكيده

اهداف: عوارض روانى جنگ مانند اختلال استرس يّ از سانحه ممكن است

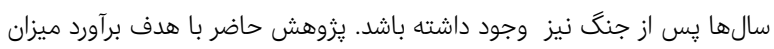

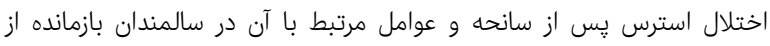

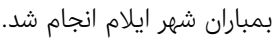

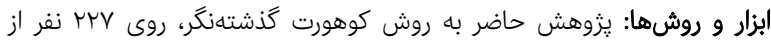

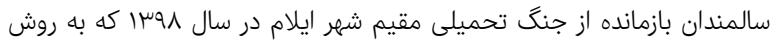

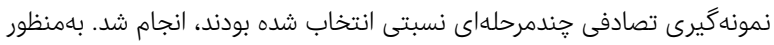

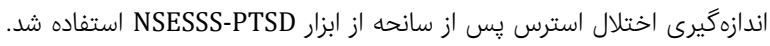

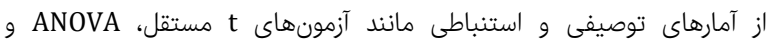

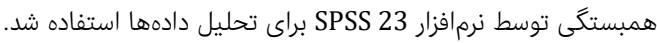

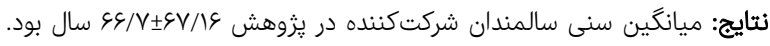

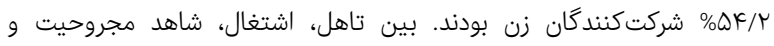

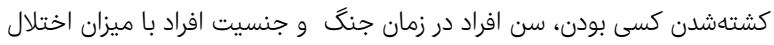

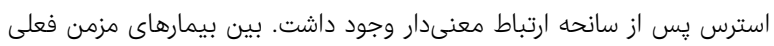

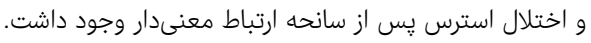

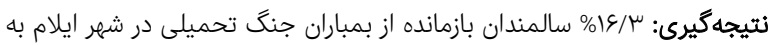

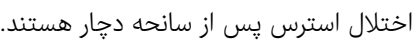
كليدوازمها: اختلال استرس يس إز إندان سانحه، سالمندان، بمباران

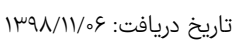

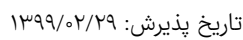

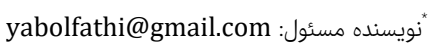

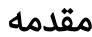

يكى از مهمترين مشكلات ناشى از جنگ براى افراد درگير با آن ابتلا به اختلال استرس يس از سانحه است كه با بيامدهاى غيرقابل بيشبينى،

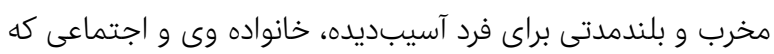

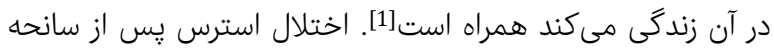
(PTSD)

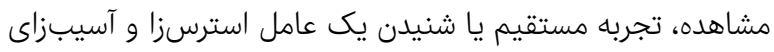

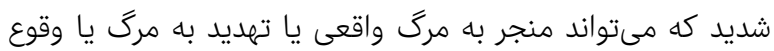

يك سانحه جدى باشد، روى مى مهديد [2].

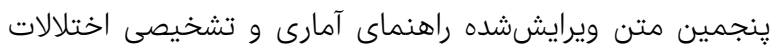

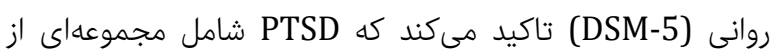

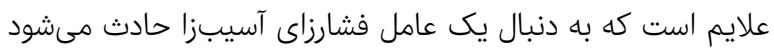




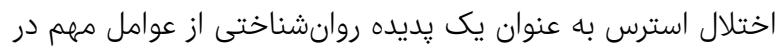

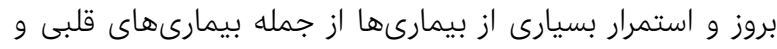

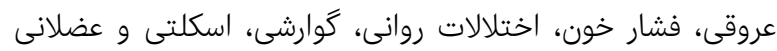

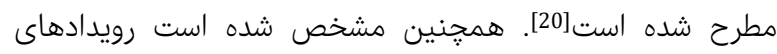

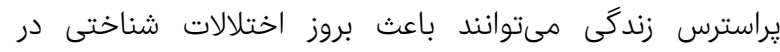

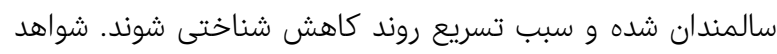

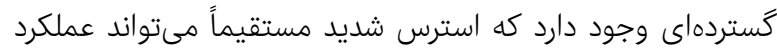

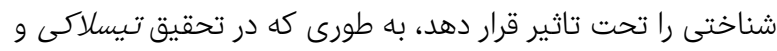

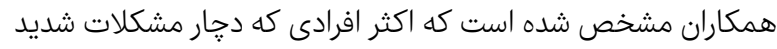

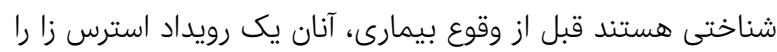

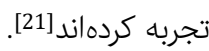

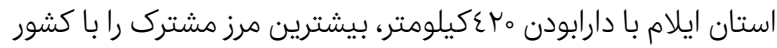

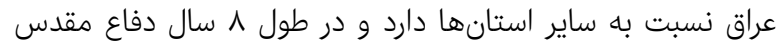

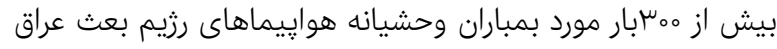

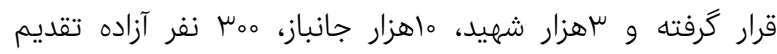
آرمانهاى مقدس جمهورى اسلامى ايران نموده است. شهر ايلام درد

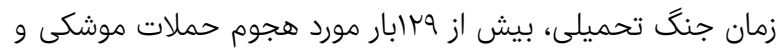

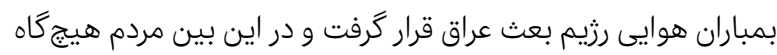

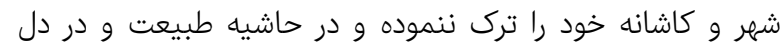

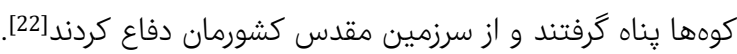

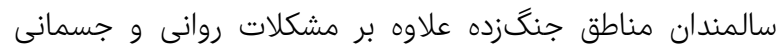

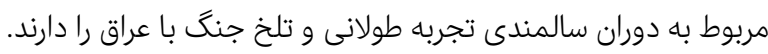

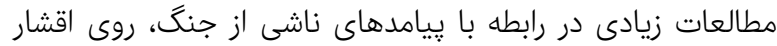

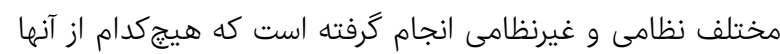

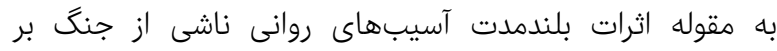

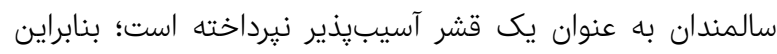

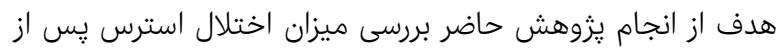
سانحه (PTSD) و عوامل مرتبط با آن در سالمندان شهر ايلام بود.

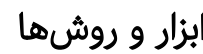

يزوهش حاضر، از نوع توصيفى و يك مطالعه كوهورت كذشتهنكر اندار

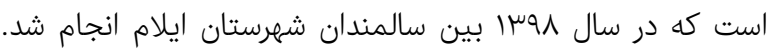

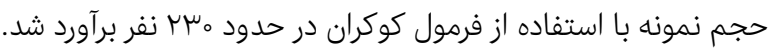

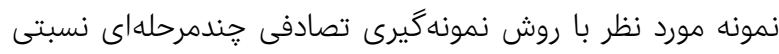

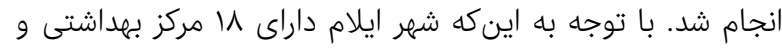

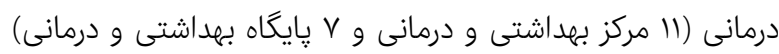

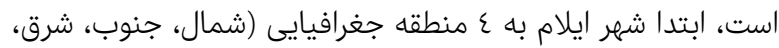

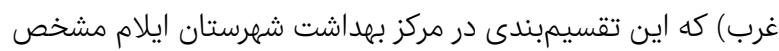

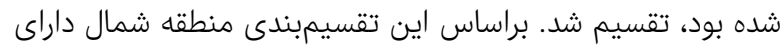

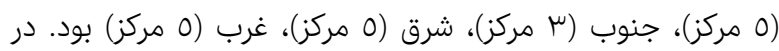

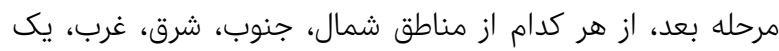

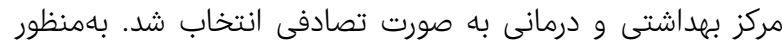
دستيابى به نمونهها از سامانه سيب استفاده شد. كليه سالمندان
ضربههاى دوران كودكى، اختلالات شخصيت، سيستم حمايتى

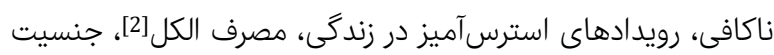

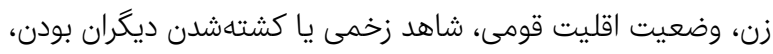

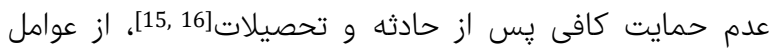

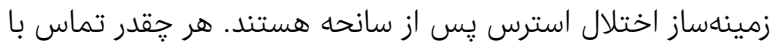

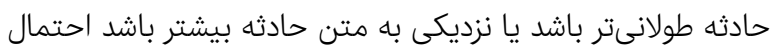

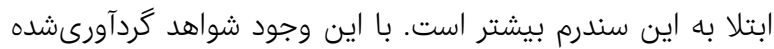

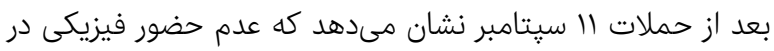

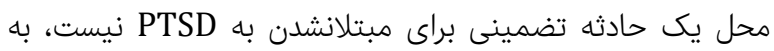

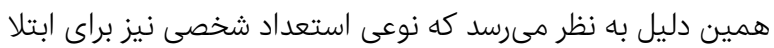

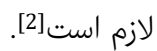
از عوارض هر جنكى، بروز مشكلات جسمى و روانى است كه براى

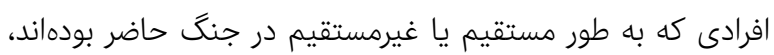

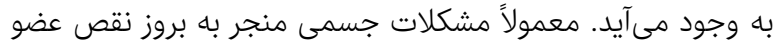

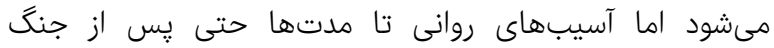

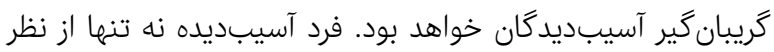

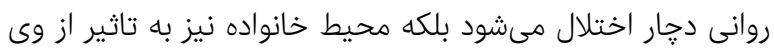

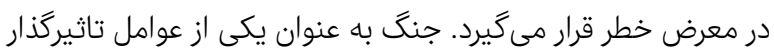

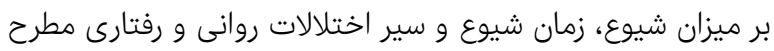

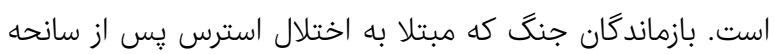

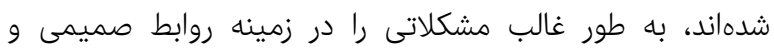
نقشهاى خانوادگى، مشكلات روانى-اجتماعى، سازَّارى خانوادئى

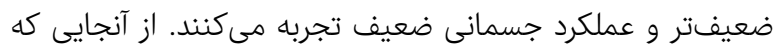
اختلال استرس يس از سانحه دربردارنده علايمى است كه ده در اعتماديف

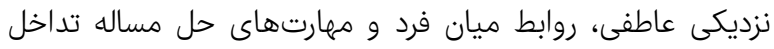

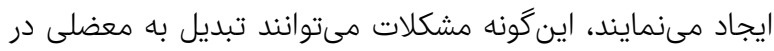

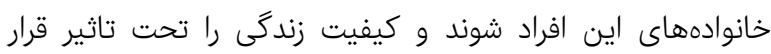
دهند[17. در مطالعهاى كه توسط كَلازمر و همكاران در سال •اه انجام شد

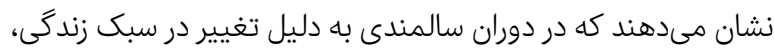

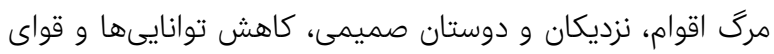

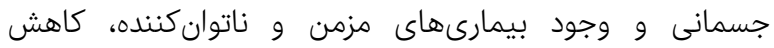

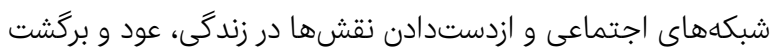

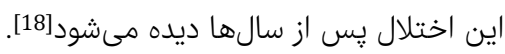

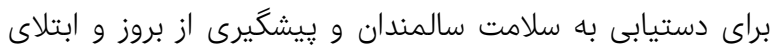

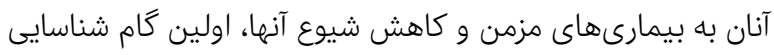

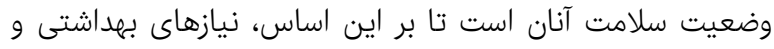

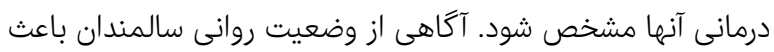

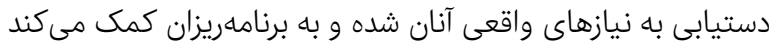

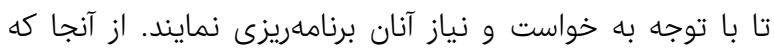

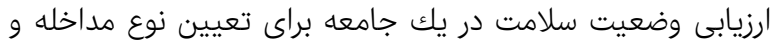

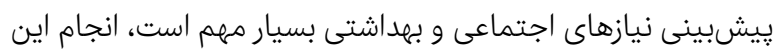

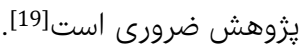


مربوط به تحصيلا حوزوى با \&/\% بود. نتايج اطلاعات

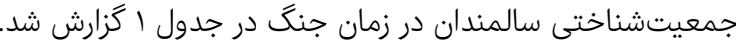

\begin{tabular}{|c|c|c|}
\hline 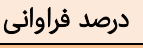 & 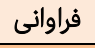 & 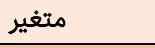 \\
\hline & & جنسيت \\
\hline$\varepsilon 0 / \wedge$ & lor & مرد \\
\hline \multirow[t]{2}{*}{$0 \varepsilon / r$} & Irr & زن \\
\hline & \multicolumn{2}{|c|}{ تحصيلات زمان جنگ } \\
\hline $79 / r$ & $10 \mathrm{~V}$ & بىسواد \\
\hline $1 \varepsilon / 1$ & $\mu r$ & ابتدايى \\
\hline $7 / 7$ & 10 & 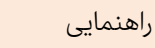 \\
\hline$\Lambda / \mu$ & 19 & 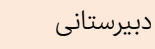 \\
\hline \multirow[t]{2}{*}{$1 / \Lambda$} & $\varepsilon$ & دانشگاهى \\
\hline & \multicolumn{2}{|c|}{ وضعيت تاهل زمان جنگ } \\
\hline $11 / 9$ & rV & مجرد \\
\hline$\wedge \mathrm{V} / \mathrm{V}$ & 199 & 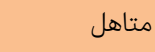 \\
\hline \multirow[t]{2}{*}{ ॰/દ } & 1 & بيوه \\
\hline & \multicolumn{2}{|c|}{ تعداد سالهاى سكونت در شهر ايلام در زمان جنگ } \\
\hline$\mu / 0$ & $\wedge$ & \\
\hline $1 / \varepsilon$ & $\mu$ & r \\
\hline$\circ / 9$ & r & $\mu$ \\
\hline \multirow[t]{2}{*}{$9 \varepsilon / \Upsilon$} & ME & 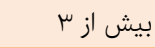 \\
\hline & \multicolumn{2}{|c|}{ 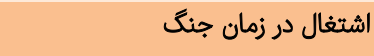 } \\
\hline $0 . / V$ & 110 & خانهدار \\
\hline$\Lambda / \Lambda$ & ro & 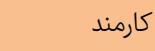 \\
\hline$r \varepsilon / V$ & 07 & آزاد \\
\hline $1 / \wedge$ & $\varepsilon$ & مستمرىبخير \\
\hline$\circ / \Lambda$ & r & بازنشسته \\
\hline $10 / 7$ & $r \varepsilon$ & كار داوطلبانه \\
\hline \multirow[t]{2}{*}{$r / 7$} & 7 & محصل \\
\hline & \multicolumn{2}{|c|}{ شاهد مجروحيت يا كشتهشدن } \\
\hline $77 / 1$ & 10. & بلى \\
\hline \multirow[t]{2}{*}{$\mu \mu / q$} & VV & خير \\
\hline & \multicolumn{2}{|c|}{ شاهد مجروحيت اعضاى خانواده } \\
\hline $17 / \mu$ & $\mu v$ & 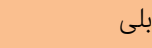 \\
\hline \multirow[t]{2}{*}{$\wedge \mu / \vee$} & 19. & خير \\
\hline & \multicolumn{2}{|c|}{ شاهد مجروحيت اقوام } \\
\hline$\mu\urcorner /\urcorner$ & $\wedge \mu$ & بلى \\
\hline $7 \mu / \varepsilon$ & $1 \varepsilon \varepsilon$ & خير \\
\hline \multicolumn{3}{|c|}{ شاهد مجروحيت غريبهها } \\
\hline $0 \varepsilon / 7$ & IrE & بلى \\
\hline$\varepsilon 0 / \varepsilon$ & $1 . \mu$ & 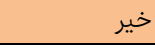 \\
\hline
\end{tabular}

ميزان اختلال استرس يس از سانحه در جمعيت مورد مطالعه

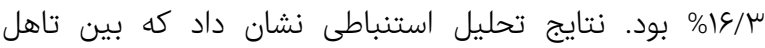

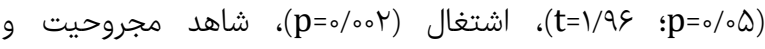

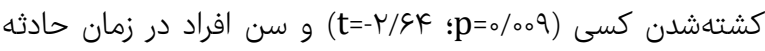

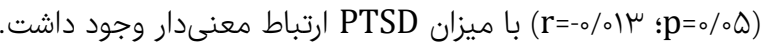

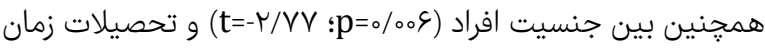

تحت يوشش هر مركز بهداشتى و درمانى براساس نام و و نام

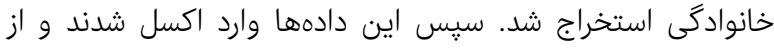

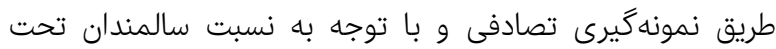
يوشش هر مركز، تعدادى سالمند انتخاب شدند. ملاكهاى بوريه ورود به به يُزوهش شامل نظامىنبودن در زمان جنگ تحميلى و در حال حاضر،

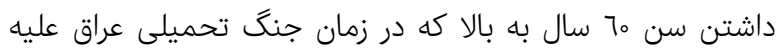
ايران در شهر ايلام زندگى كردهاند و هم اكنون ساكن ايلام هستند،

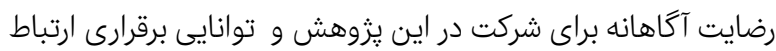

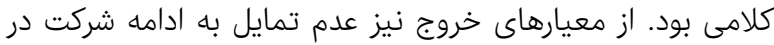
مطالعه يا حادثشدن بيمارى حاد حين انجام مصاحبه بودند.

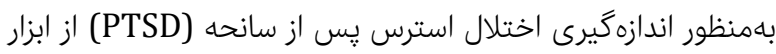
(National Stressful Events Survey NSESSS-PTSD Acute Stress Disorder Short Scale)

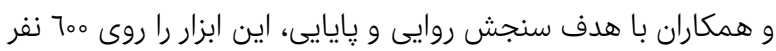

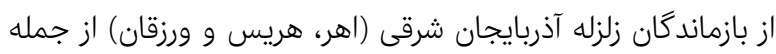

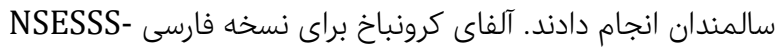
مطلوب بود ( PTSD

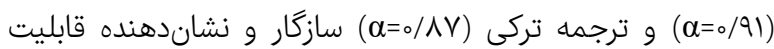

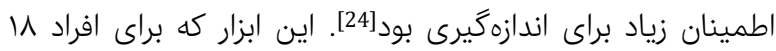

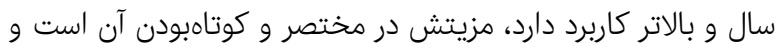

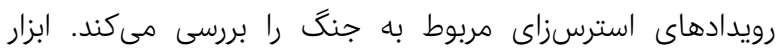
NSESSS-PTSD مجدد حادثه، علايم كرختى و اجتناب و علايم بيش برانكيختگى

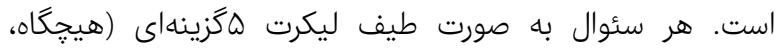
خيلىكم، به طور متوسط، زياد و به شدت) است كه به به هر يك از از

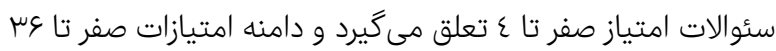

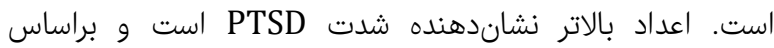

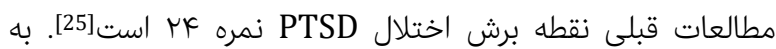

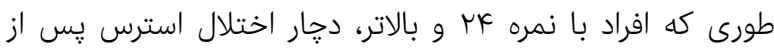

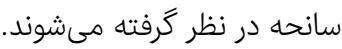
با كسب اجازه به صورت تلفنى و از طريق مراجعه حضورى به به در

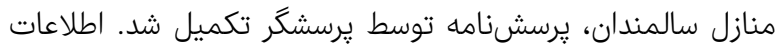

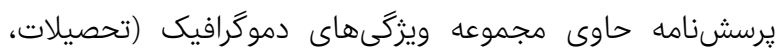
جنسيت، سن، تاهل، وضعيت ترتيبات زندگى و شغلى شعل)، ابزار

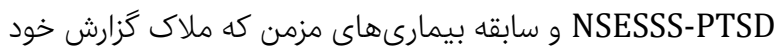

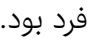
از آمارهاى توصيفى و آزمونهاى t مستقل، ANOVA و همبستخى براى سنجش عوامل مرتبط با اختلال استرس يس از سانحه توسط إمان

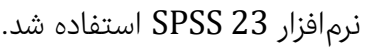

\section{يافتهها}

ميانكين سنى سالمندان شركتكننده

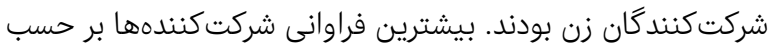

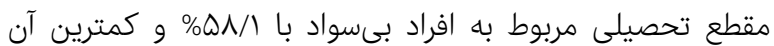


ميزان و عوامل مرتبط با اختلال استرس يس از سانحه در سالمندان بازمانده از بمباران شهر ايلام نوع مواجهه، ابزار بررسى، خصوصيت شخصيتى (براساس نظريه

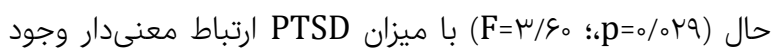

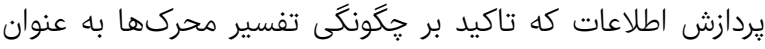

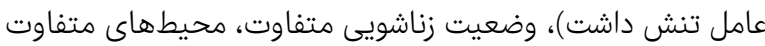

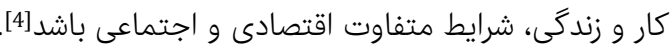
نتايج مطالعه حاضر نشان داد كه ارتباط معنىدارى بين شكل زندگى مانى

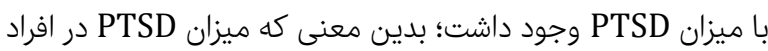

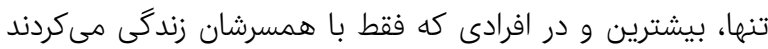

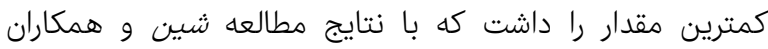

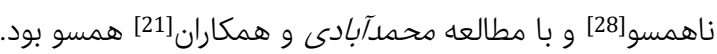
احساس تنهايى پيشبينىكننده افسردگى، كاهش فعاليت جسمى،

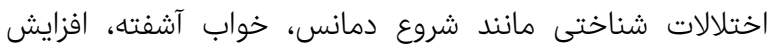

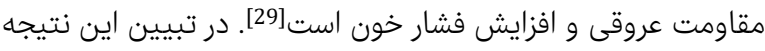

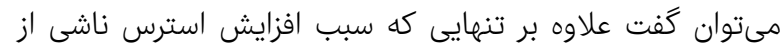
ازدستدادن حمايت اجتماعى مىشود، كشمكش بين سالمندان و ورئ

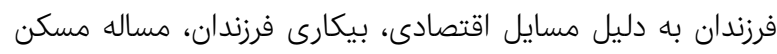
فرزندان و غيره، سبب افزايش مشكلات روحى و روانى سالمندان مىشود. از ديكر نتايج مطالعه اين بود كه ارتباط معنىدارى بين مجروحيت

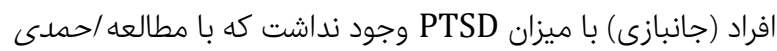

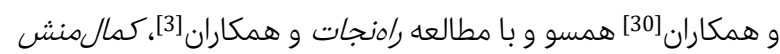

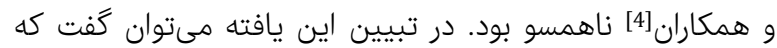

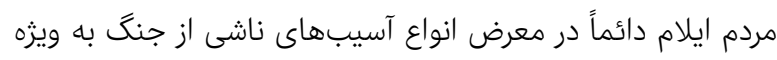

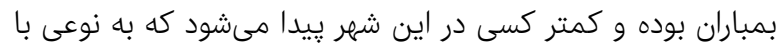

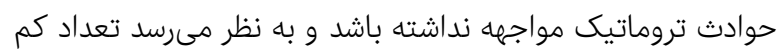

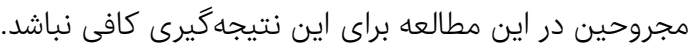

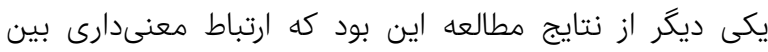

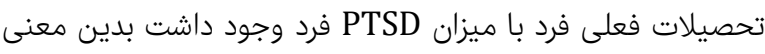

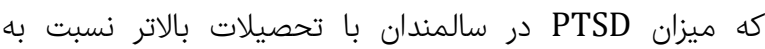

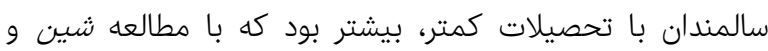

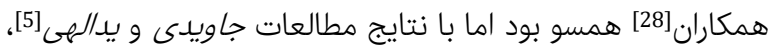
احمدى و همكاران[12]، راهنجات و همكاران [3] ناهمسو بود. در اين

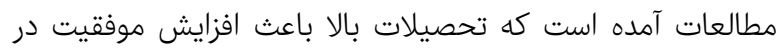
زندگى و سازگارى اجتماعى و در نتيجه كاهش ميزان

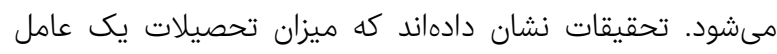

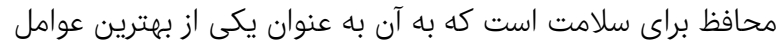

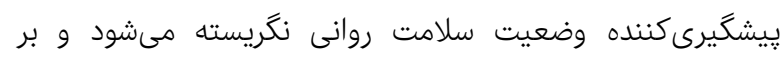

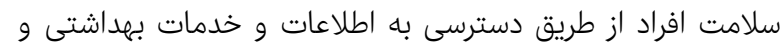

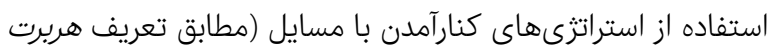

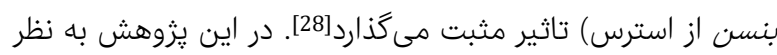

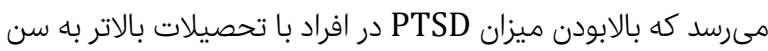

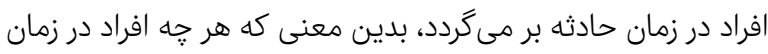
حادثه سن كمتر و شرايط مناسبترى از نظر سنى براى ادرى ادامه تحصيل داشتهاند، بيشتر تحت تاثير عوامل فشارزاى بمباران قرار كرفتنهاند. 
حداقل يك سوم بيماران مبتلا به ديابت از اختلالات خلقى رنج مىبرند

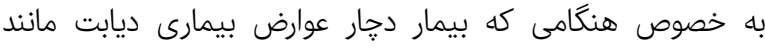
مشكلات جشم، كليه، قطع عضو و غيره شده باشند [39].

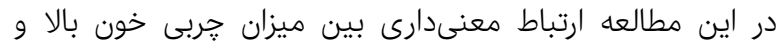

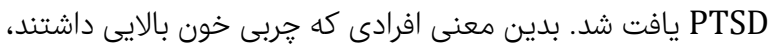

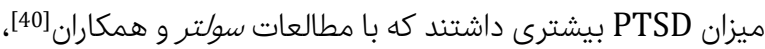
مايا و همكاران[41]، صادقى و همكاران[42] همسو بود اما با مطالعه

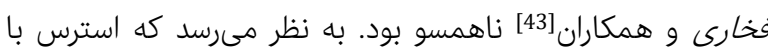

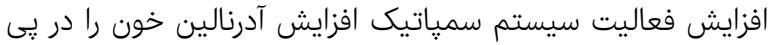

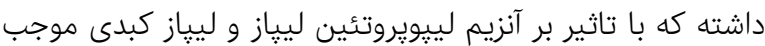
افزايش جربىهاى خون مىشود. همجنين نتايج مطالعات نشان

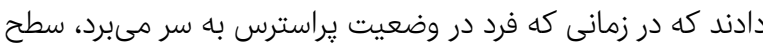

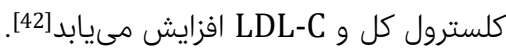
يكى ديگر از نتايج اين يزوهش ارتباط معنىدار بين اختلالات

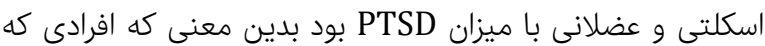
دجار اختلالات اسكلتى و عضلانى شدهاند ميزان PTSD بيشترى مئري

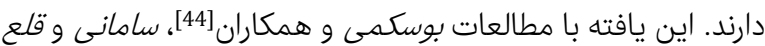

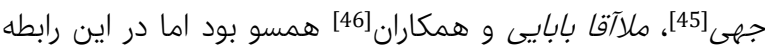

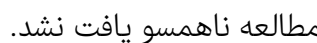

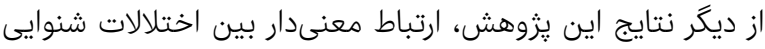

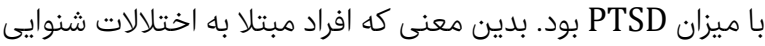

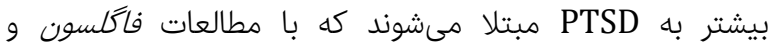
همكاران[47]، فيروزه و خالدين [48] همسو است اما مطالعه ناهمسو يافت نشد. در تبيين اين يافته مىتوان كفت كه عوامل مختلفى از جمله تحليلرفتن سلولها از نظر وزنى، حجمى و فعاليت، ضربات إنى

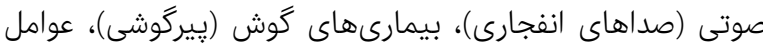
زنتيكى، رزيم غذايى، كمبود اكسيثن، كمشدن جرئن جريان خون، جمعشدن جربى در سلولها و بالارفتن كلسترول خون، بر اخدئ، اختلالات شنوايى تاثيرگذار است. از طرفى احتمال بروز ناتوانىهاى شناخ شناختى و فيزيكى از جمله شنوايى به طور قابل توجهى در سنين خيلى بالا افزايش مىيابد. كاهش شنوايى در اين دوران مىتواند منجر به به

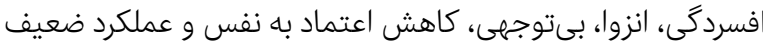

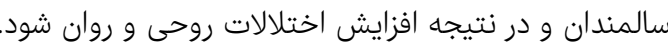

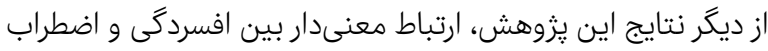

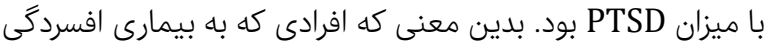

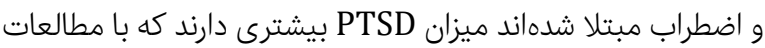

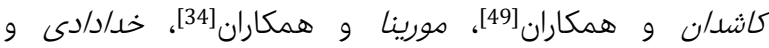

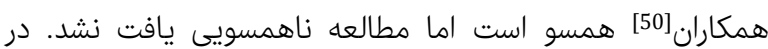
تبيين اين يافته مىتوان گفت كه افراد با دارابودن سلامت روان

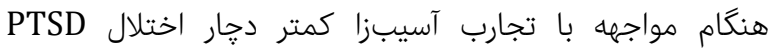
مىشوند. به سخنى ديكر سلامت روان به همراه صفات شخصيت مثبت علاوه بر آنكه مقابلهاى موثر در برابر تجارب داب آسيبزاستاست

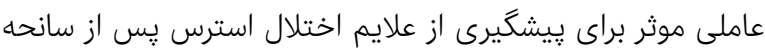

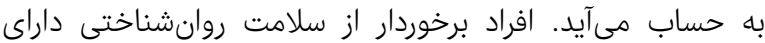

طبق نتايج اين مطالعه ميزان PTSD در زنان بيشتر از مردان بود كه

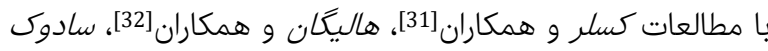

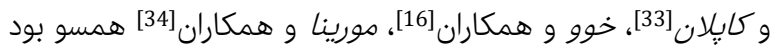

اما با مطالعه راهنجات و همكاران [3] ناهمسو بودو هوران نتايج حاصل از اين يزوهش نشان داد كه سن به به صورت معكوس

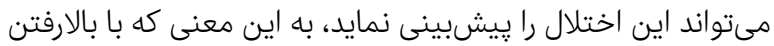

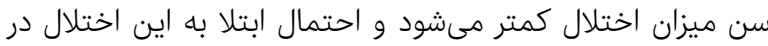

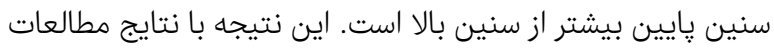

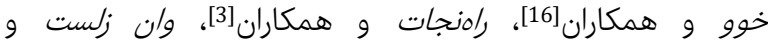
همكاران[26]، كسلر و همكاران [31]، نريمانى و همكاران [35] همسو، همات اما با مطالعه ميرى و همكاران[36] ناهمسو بود. درباره اين يافته تبيينهاى احتمالى زير را مىتوان مطرح كرد كه در تشديد اختلال همال دهران استرس يس از سانحه سن مىتواند موثر واقع شود به دليل اينكه

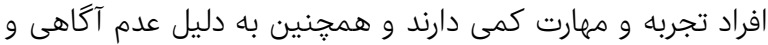

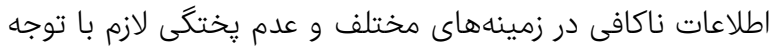
به شرايط بحرانى، مشكلات و عوارض ناشى از جنگ باعث مشكلات

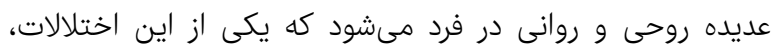
اختلال استرس يس از سانحه است.

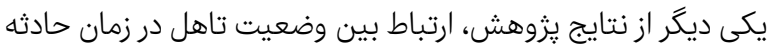
و ميزان PTSD در زمان حال بود بدين معنى كه افرادى كه در زمان رمان

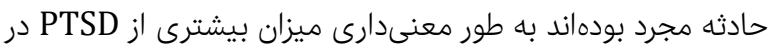

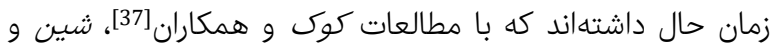
همكاران[28]، عباسى و همكاران[7] همسو بود اما با مطالعه نريمانى و همكاران[35] ناهمسو بود كه در اين مطالعه آمده بود كه افراد متاهل به دليل عوامل فشارزاى بيشتر نسبت به افراد مجرد، ميزان

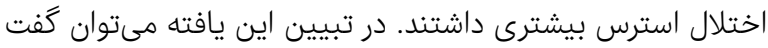

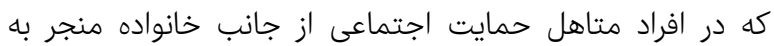

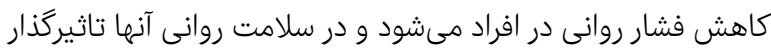

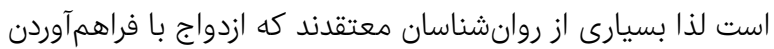
حمايت اجتماعى براى افراد منجر به حفظ بهداشت روانس روانى افراد

مىشود. يكى ديكر از نتايج يزوهش، ارتباط معنىدار بين وضعيت شاهد

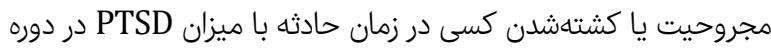

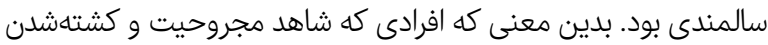

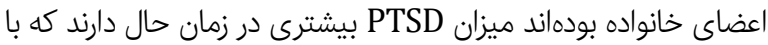

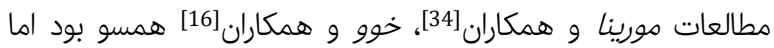
مطالعه ناهمسو يافت نشد.

از ديگر نتايج يزوهش، ارتباط ميان بيمارى ديابت با ميزان PTSD

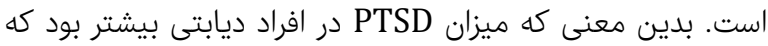

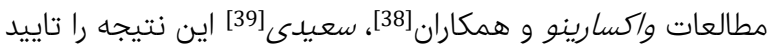
مىكنند. در اين مورد مطالعه ناهمسو يافت نشد. در كنار شواهدى

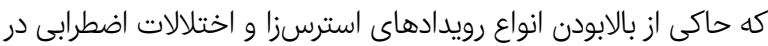

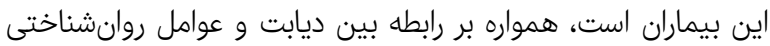

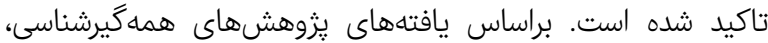


ميزان و عوامل مرتبط با اختلال استرس يس از سانحه در سالمندان بازمانده از بمباران شهر ايلام اسا

disorder (STSD) symptoms and psychological problems among devotees' wives with chronic PTSD due to war. Med Sci. 2012;22(2):122-9. [Persian]

2- Ebadi M, Azrami S, Shafighi S. Post traumatic stress disorder (PTSD). J Army Nurs Fac Iran. 2012;12(2):68-77. [Persian]

3- Rahnejat AM, Dabbaghi P, Rabiei M, Taghva A, Valipur $\mathrm{H}$, Donyavi $\mathrm{V}$, et al. Prevalence of post-traumatic stress disorder caused by war in veterans. Iran J War Public Health. 2017;9(1):15-23. [Persian]

4- Kamalmanesh A, Maredpour A. Evaluation of posttraumatic stress disorder among war survivors. Health Res J. 2017;2(2):87-98. [Persian]

5- Javidi H, Yadollahie M. Post-traumatic stress disorder. Int J Occup Environ Med. 2012;3:2-9.

6- Averill PM, Beck JG. Posttraumatic stress disorder in older adults: A conceptual review. J Anxiety Disord. 2000;14(2):133-56.

7- Abbasi Z, Mahaki B, Saberi HR. Prevalence of posttraumatic stress disorder (PTSD) among firefighters of Isfahan. Occup Med Q J. 2016;8(2):24-36. [Persian]

8- HojjatiH. Life experiences of wives of traumatized trauma victims: A phenomenological study of hermeneutics [Dissertaition]. Ahvaz: Ahvaz Jundishapur Medical and Health Services, Nursing Midwifery; 2015. [Persian]

9- Khodaie Ardakani M, Khanjani M, Mirabzadeh A, Bahmani B, Abtahi E, Sadighi G, et al. Effect of exposure to the traumatic event location in war veterans with Post Traumatic Stress Disorder. Iran J War Public Health. 2014;6(2):10-7. [Persian]

10- Raghibi M, Shirabadi A, Moallemi S, Narimani M. Demographic characteristics and post-traumatic stress disorder in prison inmates of Zahedan, Iran. J Mil Car Sci. 2016;3(1):10-7. [Persian]

11- Khodaei Ardakani MR, Khanjani MS, Mirab Zadeh A, Bahmani B, Abtahi E, Sedighi G, et al. Investigating the impact of exposure to a traumatic event place on reducing PTSD symptoms caused by war. Iran J War Public Health. 2014;22(4):10-7. [Persian]

12- Ahmadi K, Habibi M, Shahi R, Moradi A, Habibi M, Khoshkam S, et al. Research findings, limitations, and orientations in the field of veterans' mental health. J Mil Med. 2013;14(4):251-60. [Persian]

13- Koenen KC, Stellman SD, Sommer Jr JF, Stellman JM. Persisting posttraumatic stress disorder symptoms and their relationship to functioning in Vietnam veterans: A 14year followup. J Trauma Stress. 2008;21(1):49-57.

14- Rona RJ, Jones M, Iversen A, Hull L, Greenberg N, Fear NT, et al. The impact of posttraumatic stress disorder on impairment in the UK military at the time of the Iraq war. J Psychiatr Res. 2009;43(6):649-55.

15- Trepasso-Grullon E. Differences among ethnic groups in trauma type and PTSD symptom severity. Grad Stud J Psychol. 2012;14:102-12.

16- Xue C, Ge Y, Tang B, Liu Y, Kang P, Wang M, et al. A meta-analysis of risk factors for combat-related PTSD among military personnel and veterans. PloS one. 2015;10(3):e0120270.

17- Najafy M, Mohammadyfar MA, Dabiri S, Erfani N, Kamary AA. The comparison of the quality of life of the war veterans families with/without post traumatic stress disorder. Iran J War Public Health. 2011;3(3):27-35. [Persian]

18- Glaesmer H, Gunzelmann T, Braehler E, Forstmeier S, Maercker A. Traumatic experiences and post-traumatic stress disorder among elderly Germans: Results of a

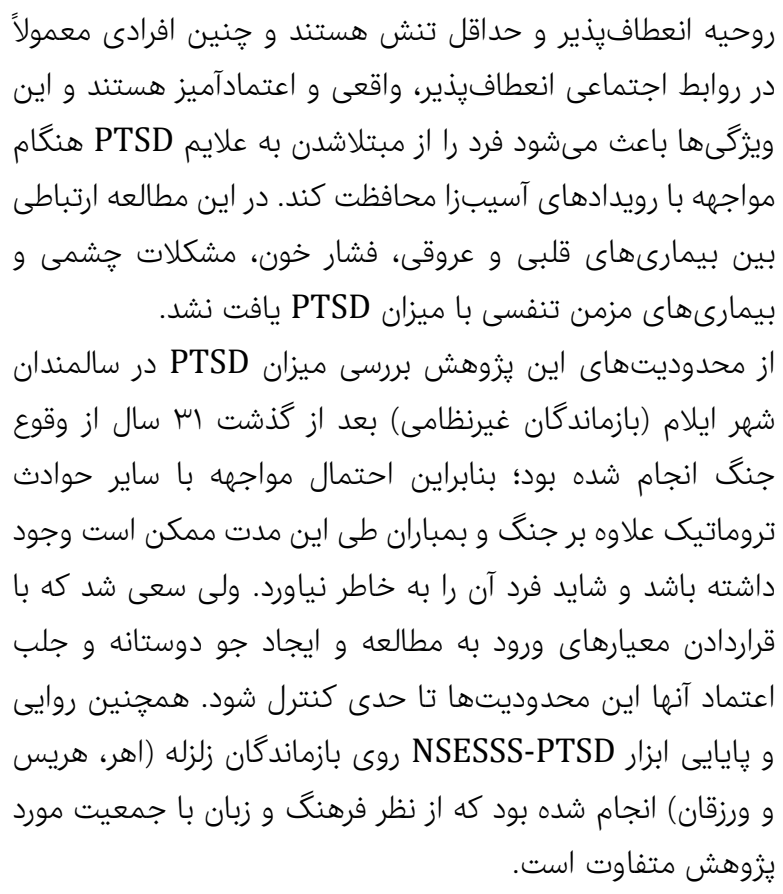

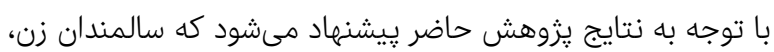

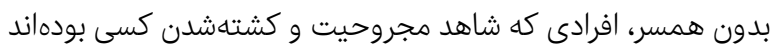

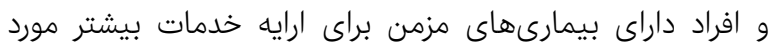

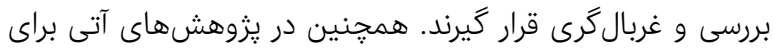

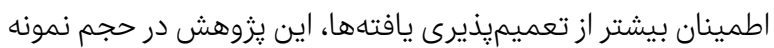

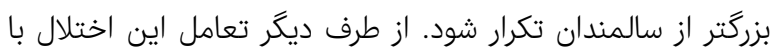

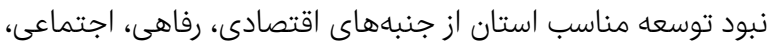

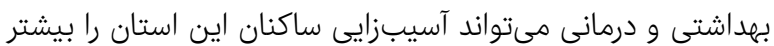
نمايد كه نيازمند توجه جدى مسئولان است.

$$
\begin{aligned}
& \text { نتيجه گيرى }
\end{aligned}
$$

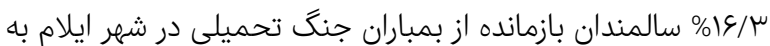

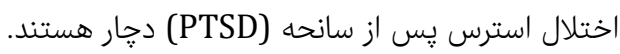

تشكر و قدردانى: از همكارى و مساعدتهاى صميمانه مسولين و

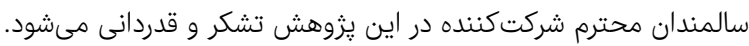

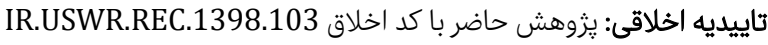

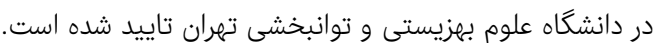

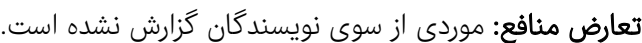

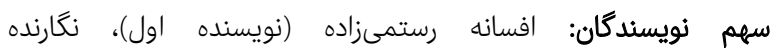

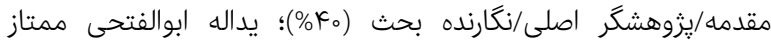

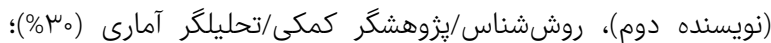
مهشيد فروغان (نويسنده سوم)، روششناس/يزوهشكًر كمكى/نگارنده

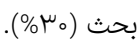
منابع مالى: موردى از سوى نويسندًان گزارش نشده است.

\section{منابع}

1- Kazemi AS, Banijamali Sh, Ahadi H, Farrokhi N. Evaluation of effectiveness of training cognitive behavioral strategies in the secondary traumatic stress 
Psychiatry. 2010;56(3):288-97.

35- Narimani M, Zahed A, Basharpoor S. Prevalence of posttraumatic stress disorder in hospital emergency nurses and fire department workers in Uremia city. J Res Behav Sci. 2010;8(1):69-74. [Persian]

36- Miri MR, Salehiniya H, Tiyuri A, Bahlgerdi M, Taghizadeh AA. Prevalence of mental disorders and its related factors among elderly of Birjand, 2014. J Geriatr Nurs. 2016;2(2):94-103. [Persian]

37- Cook JM, Riggs DS, Thompson R, Coyne JC, Sheikh JI. Posttraumatic stress disorder and current relationship functioning among world war II ex-prisoners of war. J Fam Psychol. 2004;18(1):36-45.

38- Vaccarino V, Goldberg J, Rooks C, Shah AJ, Veledar E, Faber TL, et al. Post-traumatic stress disorder and incidence of coronary heart disease: A twin study. J Am Coll Cardiol. 2013;62(11):970-8.

39- Saeidi P. Effectiveness of biofeedback-relaxation on depression, anxiety and blood glucose in type i diabetic patients. J Clin Psychol. 2016;8(4):7.

40- Solter V, Thaller V, Karlovic D, Crnkovic D. Elevated serum lipids in veterans with combat-related chronic posttraumatic stress disorder. Croat Med J. 2002;43(6):685-9.

41- Maia DB, Marmar CR, Mendlowicz MV, Metzler T, Nóbrega A, Peres MC, et al. Abnormal serum lipid profile in Brazilian police officers with post-traumatic stress disorder. J Affect Disord. 2008;107(1-3):259-63.

42- Sadgi M, Roafza HR, Yazdga S, Khani A, Aadgan NS. Is stress levels linked to impaired blood lipids? Esfahan healthy heart program. Behav Sci Res. 2012;9(5):382-8. [Persian]

43- Fakhari A, Ebrahimzadeh M, Shiva S, Fekrat S, Mohammadpoorasl A. Effect of mental stress on serum triglyceride level. Res J Biol Sci. 2007;2(4):476-8.

44- Buscemi V, Chang W-J, Liston MB, McAuley JH, Schabrun S. The role of psychosocial stress in the development of chronic musculoskeletal pain disorders: Protocol for a systematic review and meta-analysis. Syst Rev. 2017;6(1):224.

45- Samani S, Ghaljahi M. Relationship between the prevalence of musculoskeletal disorders and occupational stress among carpet weavers in Sistan and Baluchestan Province, Iran, in 2017. J Health Res Commun. 2018;4(2):23-32. [Persian]

46- Molla Agha Babaei AH, Yazdi M, Karimi Zeverdegani S, Barakat S. Prevalence of musculoskeletal disorders and its relationship with occupational stress among workers at a steel industry. Iran Occup Health. 2016;13(3):63-72. [Persian]

47- Fagelson MA. The association between tinnitus and posttraumatic stress disorder. Am J Audiol. 2007;16(2):107-17.

48- Firozeh M, Khaledian Z. Identifying Physical health status and its association with depression among retired staffs of Bojnourd education department. J Geriatr Nurs. 2015;1(3):104-13. [Persian]

49- Kashdan TB, Morina N, Priebe S. Post-traumatic stress disorder, social anxiety disorder, and depression in survivors of the Kosovo War: Experiential avoidance as a contributor to distress and quality of life. J Anxiety Disord. 2009;23(2):185-96.

50- Khodadadi N, Ghanbari Khanghah A, Mousavi SM, Khaleghdoos T, Moosavi MJ. Related factors to onset of post-traumatic stress disorder after road accidents. J Holist Nurs Midwifery. 2014;24(2):9-17. [Persian] representative population-based survey Psychogeriatr. 2010;22(4):661-70.

19- Barati M, Fathi Y, Soltanian A, Moeini B. Mental health condition and health promoting behaviors among elders in Hamadan. Avicenna J Nurs Midwifery Care. 2012;20(3):12-22. [Persian]

20- Azizi M, Gavanmard GH. Stress management, history and theories. National Conference on New World Achievements in Education, Psychology, Law and SocioCultural Studies, Zaraghan Branch of Islamic Azad University, 2018. Tehran: Civilica; 2018. [Persian]

21- Shiri Mohammadabadi A, Yazdkhasti F, Dadgari A. Determining the major stressful events in elderly people and their relation with depession and cognitive decline. TOLOOE BEHDASHT. 2015;13(6):139-51. [Persian]

22- Sahraei M. History of Defa-e-Moqadas in Ilam Province, 1980-1988. Tehran: Fatahan; 2011. [Persian]

23- LeBeau R, Mischel E, Resnick H, Kilpatrick D, Friedman M, Craske M. Dimensional assessment of post traumatic stress disorder in DSM-5. Psychiatry Res. 2014;218(1-2):143-7.

24- Rafiey H, Alipour F, LeBeau R, Salimi Y, Sayad M. Evaluating the Persian translation of the national stressful events survey PTSD short scale in a sample of Iranian Earthquake Survivors. J Loss Trauma. 2017;22(8):660-8. 25- Evren C, Dalbudak E, Aydemir 0, Koroglu E, Evren B, Ozen S, et al. Psychometric properties of the Turkish PTSD-Short Scale in a sample of undergraduate students. Klinik Psikofarmakoloji Bülteni-Bulletin Clin Psychopharmacol. 2016;26(3):294-302.

26- Van Zelst WH, de Beurs E, Beekman AT, Deeg DJ, van Dyck R. Prevalence and risk factors of posttraumatic stress disorder in older adults. Psychother Psychosom. 2003;72(6):333-42.

27- Knaevelsrud C, Böttche M, Pietrzak RH, Freyberger HJ, Renneberg B, Kuwert P. Integrative testimonial therapy: An Internet-based, therapist-assisted therapy for German elderly survivors of the world war II with posttraumatic stress symptoms. J Nerv Ment Dis. 2014;202(9):651-8.

28- Shin SM, Kim HJ, Liw L, Kim S. Depression and PTSD in Pashtun women in Kandahar, Afghanistan. Asian Nurs Res. 2009;3(2):90-8.

29- Khodabakhshi-Koolaee A, Froozani AF. A comparison between loneliness and death anxiety among active and inactive elderly male living in nursing homes in Yazd. Nurs J Vulnerable. 2018;5(16):1-15. [Persian]

30- Ahmadi K, Reshadatjoo M, Karami GR. Evaluation of PTSD in Sardasht survivors of chemical warfare. Urmia Med J. 2010;21(1):1-9. [Persian]

31- Kessler RC, Rose S, Koenen KC, Karam EG, Stang PE, Stein DJ, et al. How well can post-traumatic stress disorder be predicted from pre-trauma risk factors? An exploratory study in the WHO World Mental Health Surveys. World Psychiatry. 2014;13(3):265-74.

32- Halligan R, Whitburn SC. Mental pathology based on clinical perspectives on mental disorders DSM-IV-TR. Seyed Mohammadi Y, translator. Tehran: Ravan Publisher; 2005. [Persian]

33- Sadock BJ, Sadock VA. Kaplan \& Sadok psychiatric summary: Based on DSM-5. New York City: Lippincott Williams \& Wilkins; 2019.

34- Morina N, Rudari V, Bleichhardt G, Prigerson HG. Prolonged grief disorder, depression, and posttraumatic stress disorder among bereaved Kosovar civilian war survivors: A preliminary investigation. Int J Soc 